\title{
Prognostic Value of Venous Thromboembolism Risk Assessment Models in Patients with Severe COVID-19
}

\author{
Luis H. Paz Rios ${ }^{1}$ Iva Minga ${ }^{1}$ Esther Kwak ${ }^{2}$ Ayman Najib ${ }^{2}$ Ashley Aller ${ }^{2}$ Elizabeth Lees ${ }^{2}$ \\ Victor Macrinici ${ }^{2}$ Kaveh Rezaei Bookani ${ }^{1}$ Amit Pursnani ${ }^{1}$ Joseph Caprini ${ }^{3}$ Alex C. Spyropoulos ${ }^{4}$ \\ Alfonso Tafur ${ }^{1}$
}

${ }^{1}$ Cardiovascular Division, Department of Medicine, NorthShore University Health System, Evanston, Illinois, United States

2 Department of Medicine, NorthShore University Health System, Evanston, Illinois, United States

3 Pritzker School of Medicine, University of Chicago, Chicago, Illinois, United States

${ }^{4}$ Department of Medicine, Donald and Barbara Zucker School of Medicine at Hofstra/Northwell Anticoagulation and Clinical Thrombosis Services, Northwell Health at Lenox Hill Hospital, NY, NY, United States

\begin{abstract}
Address for correspondence Luis H. Paz Rios, MD, Cardiovascular Division, Department of Medicine, NorthShore University Health System, 2650 Ridge Avenue, Evanston, IL 60201, United States (e-mail: pazrioslh@gmail.com).
\end{abstract}

TH Open 2021;5:e211-e219.

\begin{abstract}
Keywords

- COVID-19

- risk assessment

- thrombosis

- mortality predictor

- IMPROVE score

- Caprini score

Introduction Severe novel corona virus disease 2019 (COVID-19) causes dysregulation of the coagulation system with arterial and venous thromboembolism (VTE). We hypothesize that validated VTE risk scores would have prognostic ability in this population.

Methods Retrospective observational cohort with severe COVID-19 performed in NorthShore University Health System. Patients were $>18$ years of age and met criteria for inpatient or intensive care unit (ICU) care. The International Medical Prevention Registry on Venous Thromboembolism (IMPROVE) and Caprini scores were calculated and patients were stratified.

Results This study includes 184 patients, mostly men (63.6\%), Caucasian (54.3\%), 63 years old (interquartile range [IQR]: $24-101$ ), and $57.1 \%$ of them required ICU care. Twenty-seven (14.7\%) thrombotic events occurred: 12 (6.5\%) cases of disseminated intravascular coagulation (DIC), 9 (4.9\%) of pulmonary embolism, 5 (2.7\%) of deep vein thrombosis, and 1 (0.5\%) stroke. Among them, 86 patients (46.7\%) died, 95 (51.6\%) were discharged, and 3 (1.6\%) were still hospitalized. "Moderate risk for VTE" and "High risk for VTE" by IMPROVE score had significant mortality association: (hazard ratio [HR]: 5.68; 95\% confidence interval $[\mathrm{Cl}]: 2.93-11.03 ; p<0.001)$ and $(\mathrm{HR}=6.22 ; 95 \% \mathrm{Cl}: 3.04-12.71$; $p<0.001$ ), respectively, with $87 \%$ sensitivity and $63 \%$ specificity (area under the curve $[A U C]=0.752, p<0.001)$. "High Risk for VTE" by Caprini score had significant mortality association ( $\mathrm{HR}=17.6$; $95 \% \mathrm{Cl}$ : 5.56-55.96; $p<0.001)$ with $96 \%$ sensitivity and $55 \%$ specificity ( $A U C=0.843, p<0.001$ ). Both scores were associated with thrombotic events when classified as "High risk for VTE" by IMPROVE (HR $=6.50$; $95 \% \mathrm{Cl}$ : $2.72-15.53$; $p<0.001)$ and Caprini scores (HR=11.507; 95\% Cl: 2.697-49.104; $p=0.001)$.

Conclusion The IMPROVE and Caprini risk scores were independent predictors of mortality and thrombotic events in severe COVID-19. With larger validation, this can be useful prognostic information.
\end{abstract}

received

February 11, 2021

accepted after revision

April 9, 2021
DOI https://doi.org/

$10.1055 / \mathrm{s}-0041-1730293$.

ISSN 2512-9465. (c) 2021. The Author(s).

This is an open access article published by Thieme under the terms of the Creative Commons Attribution License, permitting unrestricted use, distribution, and reproduction so long as the original work is properly cited. (https://creativecommons.org/licenses/by/4.0/)

Georg Thieme Verlag KG, Rüdigerstraße 14, 70469 Stuttgart, Germany 


\section{Introduction}

Initially described in China, the novel coronavirus disease 2019 (COVID-19) pandemic is caused by the severe acute respiratory syndrome-coronavirus- 2 (SARS-CoV-2) ${ }^{1}$ and has resulted in significant morbidity and mortality worldwide with approximately 1,600,000 deaths as of December 15 , $2020,{ }^{2}$ constituting an urgent threat to global health.

Most affected individuals with COVID-19 will present a milder and self-limited form of the disease with flu-like symptoms. ${ }^{3}$ However, a smaller portion will develop severe or critical illness and potentially acute respiratory distress syndrome, systemic inflammatory response syndrome, and multiorgan failure with associated mortality in up to $50 \%$ of cases. $^{4}$

It is known that hospitalized patients with acute medical illness are at increased risk for the development of venous thromboembolism (VTE). ${ }^{5}$ In addition to this, there is a growing body of evidence showing the direct and indirect endothelial damage that occurs as a consequence of COVID-19, further predisposing patients to thrombotic disease in the venous and the arterial circulation., 6,7

As the number of cases of COVID-19 steadily increases, tools with the ability to risk stratify and triage these affected individuals can help allocate resources and optimize medical care. Several risk factors for disease severity and poorer outcomes have been identified ${ }^{1,8,9}$; however, controversy remains in the optimal way to classify these patients. ${ }^{10}$ Considering that the particular endothelial involvement and the high incidence of VTE in patients with COVID-19 are highly associated with worse outcomes, ${ }^{11}$ we hypothesized that current validated and readily available VTE risk assessment models (RAM) like the International Medical Prevention Registry on Venous Thromboembolism (IMPROVE) and Caprini scores, would provide prognostic information and predict mortality in patients with severe COVID-19 disease. ${ }^{12,13}$

\section{Methods}

\section{Study Patients}

This was a retrospective observational study of consecutive hospitalized patients with severe COVID-19 disease, performed in NorthShore University Health System from March 12, 2020, through July 30, 2020. Patients were at least 18 years of age with SARS-CoV-2 infection confirmed by polymerase chain reaction. Severe COVID-19 disease was defined by the presence of with dyspnea, hypoxia (peripheral saturation $<92 \%$, need for oxygen supplementation, or significant lung involvement on imaging. All patients met criteria for inpatient-level care (IC) or intensive care unit (ICU)-level care when they required invasive ventilatory support.

On April 15, an institutional thromboprophylaxis protocol for patients with COVID-19 was implemented in line with multisociety recommendations. ${ }^{14}$ All patients underwent individualized VTE risk stratification with the IMPROVE score or Caprini score. Thromboprophylaxis with low molecular weight heparin (LMWH) at prophylactic or intermediate doses (i.e., enoxaparin $40 \mathrm{mg}$ sub cutaneous daily or $40 \mathrm{mg}$ s.c. twice daily if patients met criteria: body mass index [BMI] $>30 \mathrm{~kg} / \mathrm{m}^{2}$, need for high-flow nasal cannula, mechanical ventilation, sepsis-induced coagulopathy score of $\geq 4$, and D-dimer $>6$ times upper limit of normal) was the preferred treatment modality, unless patients had severe renal insufficiency. Furthermore, thromboprophylaxis was extended for 2 weeks with LMWH for patients over 40 years of age with reevaluation of VTE risk at follow-up. Alternatively, extended thromboprophylaxis up to 45 days with rivaroxaban $10 \mathrm{mg}$ daily was recommended when criteria were met (over 50 years old, IMPROVE VTE risk $\geq 4$, Caprini score $>8$, D-dimer $>1 \mathrm{mg}$ / $\mathrm{dL}$, ICU stay, prior VTE, active cancer, and paralysis).

The present study was approved by the local Institutional Review Board (Federal Wide Assurance: FWA00003000) and written consent was waived given its retrospective design. The database with deidentified patient information was set up and maintained by the Cardiovascular Division. ${ }^{15}$

\section{Data Collection}

Faculties of the Department of Internal Medicine and the Division of Cardiology settled and extracted data by review of the institutional electronic medical record. We abstracted demographic data including age, gender, BMI, and laboratory parameters at hospitalization. Comorbidities, defined as simultaneous presence of $>1$ chronic condition, were recorded on hospital admission.

Laboratory data were collected within the first 5 days from hospitalization and, taking into consideration, the available peak values.

The IMPROVE and Caprini scores were calculated at the time of data collection and by a second author at the time of data analysis for corroboration. Patients were classified according to the IMPROVE risk score in "Low risk for VTE" (score 0-1), "Moderate risk for VTE" (score 2-3), and "High risk for VTE" (score $\geq 4$ ). ${ }^{16,17}$

For the Caprini score, patients were classified according to their risk scores and dichotomized into "Low to Moderate risk for VTE" (score 0-4) and "High Risk for VTE" (score $\geq 5$ ) to make groups comparable, and were subsequently stratified by Caprini score at accepted cut-offs as follows: very low risk (Caprini score: $0-2$ ), moderate risk (Caprini score: 3-4), high risk (Caprini score 5-6), very high risk (Caprini score: 7-8), and the highest risk (Caprini score $>8) .{ }^{18,19}$

\section{Outcomes}

VTE was defined as deep vein thrombosis (DVT) or pulmonary embolism (PE) when clinically observed and treated during hospitalization. Patients were considered to have DVT if treated for DVT and/or had positive compression ultrasonography test. Routine screening for DVT was not instituted by the time of adjudication, this was in line with the recommendations from the World Health Organization until the time of censoring. Patients were considered to have PE if they were treated for PE and had a positive lung scan, pulmonary angiogram, or spiral computed tomography (CT) scan. The International Society for Thrombosis and 
Hemostasis (ISTH) criteria for the diagnosis of disseminated intravascular coagulation (DIC) was used.

Clinical and mortality outcomes were extracted and censored at the time of death and at time of last follow-up until July 30, 2020. The data and scores were verified by a second independent author for their accuracy and, in case of discrepancy, adjusted by consensus.

\section{Statistical Analysis}

Descriptive data were summarized to characterize the distribution of baseline parameters. Kolmogorov-Smirnov and Shapiro-Wilk tests were used to evaluate if continuous variables were normally distributed; we used Student's $t$-test to compare normally distributed continuous variables and Mann-Whitney $U$-test to compare nonparametric continuous variables; Pearson's Chi-square test or Fisher's exact test were used to compare categorical variables.

We performed univariate and multivariate analyses using Cox's proportional hazards regression with forward modeling to explore the association of each variable and mortality, as well as thrombotic outcomes. Variables with statistical significance in univariate analysis $(p<0.05)$ were included in multivariate analysis to identify independent risk factors for mortality and thrombotic outcomes. Development of a thrombotic outcomes was treated as a time-dependent variable to eliminate the effect of misclassification from immortal time bias.

We used the receiver-operating characteristics (ROCs) curve to analyze the discriminatory capacity of each RAM, and used the Kaplan-Meier method to estimate the cumulative event-free survival curves for the different groups.

We present categorical variables as absolute numbers and percentages, and continuous variables by their mean and standard deviation, or by the median and interquartile range (IQR) as appropriate. All statistical analyses were performed with SPSS version 27.0 (IBM, Armonk, New York, United States). A $p$-value of $<0.05$ was considered statistically significant.

\section{Results}

A total of 184 consecutive patients with severe COVID-19 were included for analysis. The patients in this study were predominantly men (63.6\%), Caucasian (54.3\%), 63 years of age (IQR: 24-101 years), and the majority (57.1\%) required ICU-level care. Patients were hospitalized on average 1 day (IQR: 0-25 days) after diagnosis of COVID-19, spent on average 7 days (IQR: 0-68 days) in the hospital, and were followed-up for a median of 52 days (IQR: 0-108 days) from their initial encounter.

At the time of censoring, a total of 27 (14.7\%) thrombotic events had occurred. There were 12 (6.5\%) cases of DIC, 9 (4.9\%) cases of pulmonary embolism, 5 (2.7\%) DVT, and $1(0.5 \%)$ stroke. A total of 86 patients (46.7\%) died, 95 patients (51.6\%) were discharged, and 3 patients (1.6\%) were still hospitalized and undergoing medical care at the time of censoring.

Comparison between the groups showed that mortality was significantly higher in patients who were older, had hypertension, diabetes mellitus, coronary artery disease, cerebrovascular disease, cancer, and patients who required ICU-level care.
Mortality was significantly higher in those classified as "Moderate risk for VTE" and "High risk for VTE" by the IMPROVE score, and "High risk for VTE" by the Caprini score (- Table $\mathbf{1}$ ).

Direct comparison of laboratory values collected revealed that nonsurvivors had significantly lower hemoglobin and albumin levels. Also, nonsurvivors had significantly higher prothrombin time, international normalized ratio (INR), D-dimer levels, creatinine levels, C-reactive protein levels, aspartate transaminase, and significantly higher absolute lymphocyte count ( - Table 2 ).

\section{The IMPROVE Score and Mortality}

In univariate analysis, patients classified by the IMPROVE score as "Moderate risk for VTE" and "High risk for VTE" had a significant association with mortality with univariate hazard ratio $(\mathrm{HR})=2.02$; 95\% confidence interval $(\mathrm{CI})$ : 1.27-3.21; $p=0.003$, and $\mathrm{HR}=2.49 ; 95 \% \mathrm{CI}: 1.62-3.83 ; p<0.001$, respectively. When adjusted for other variables in multivariate Cox's regression analysis, the association remained significant for "High risk for VTE" (HR=6.22; 95\% CI: 3.04-12.71; $p<0.001)$, followed by "Moderate risk for VTE" (HR $=5.68$; 95\% CI: 2.93$11.03 ; p<0.001)$, the presence of diabetes $(\mathrm{HR}=1.70 ; 95 \% \mathrm{Cl}$ : $1.10-2.63 ; p=0.016)$, and hypertension ( $\mathrm{HR}=1.63 ; 95 \% \mathrm{CI}$ : $1.02-2.59 ; p<0.040)$. An ROC curve was plotted with an area under the curve (AUC) of $0.752(p<0.001)$ and a corresponding sensitivity of $87 \%$ and specificity of $63 \%$ for the patients classified as "Moderate risk for VTE" or above (-Fig. 1A). When stratified by IMPROVE scores, the cumulative mortality increased in a linear fashion with increasing score. Patients classified at "Low risk for VTE" had an in-hospital mortality of $15.1 \%$, "Moderate risk for VTE" had a mortality of $66.7 \%$, and "High risk for VTE" had a mortality of $68.1 \%$ (-Fig. 2A).

\section{Caprini Risk Assessment Model and Mortality}

In univariate analysis, patients classified by the Caprini RAM as "High Risk for VTE" had a significant association with mortality $(\mathrm{HR}=18.6$; 95\% CI: 5.87-59.06; $p<0.001)$. Furthermore, this association remained significant when adjusted in multivariate Cox's regression analysis ( $\mathrm{HR}=17.6$; 95\% CI: 5.56-55.96; $p<0.001$ ), followed by the presence of diabetes ( $\mathrm{HR}=1.60$; 95\% CI: $1.05-2.46 ; p=0.029)$. An ROC curve was plotted with an AUC of $0.843(p<0.001)$ and a corresponding sensitivity of $96 \%$ and specificity of $55 \%$ for the designated dichotomized cutoff ( - Fig. 3A).

When stratified by Caprini scores, the cumulative mortality increased in a linear fashion with increasing score. Patients classified at very low risk (Caprini score: $0-2$ ) had no mortality events. Those stratified at moderate risk (Caprini score: $3-4$ ) had $6.8 \%$ mortality, high risk (Caprini score: $5-6$ ) had a $44.4 \%$ mortality, very high risk (Caprini score: 7-8) had a $60.8 \%$ mortality, and the highest risk (Caprini score $>8$ ) had a mortality rate of $80 \%$ ( - Fig. 4 A).

\section{Venous Thromboembolism Risk Assessment Models in Prediction of Thrombotic Events}

In univariate and multivariate analyses, both scores were statistically associated with thrombotic event occurrence when classified as "High risk for VTE" by IMPROVE score 
e214 Prognostic Value of VTE Risk Assessment Models Paz Rios et al.

Table 1 Epidemiologic characteristics of critically ill patients with COVID-19, NorthShore University Health System

\begin{tabular}{|c|c|c|c|c|c|c|c|}
\hline \multirow[t]{2}{*}{ Variables } & \multicolumn{2}{|c|}{ Cohort $(n=184)$} & \multicolumn{2}{|c|}{ Alive $(n=98)$} & \multicolumn{2}{|c|}{ Deceased $(n=86)$} & \multirow[t]{2}{*}{${ }^{c} p$-Value } \\
\hline & $\begin{array}{l}\text { Mean/ } \\
\text { median/n }\end{array}$ & SD/IQR/\% & $\begin{array}{l}\text { Mean/ } \\
\text { median/n }\end{array}$ & $\mathrm{SD} / \mathrm{IQR} / \%$ & $\begin{array}{l}\text { Mean/ } \\
\text { median/n }\end{array}$ & $\mathrm{SD} / \mathrm{IQR} / \%$ & \\
\hline${ }^{\mathrm{a}}$ Age (y) & 63 & $24-101$ & 59 & $24-101$ & 67.5 & $37-91$ & 0.003 \\
\hline${ }^{\mathrm{b}} \mathrm{Age} \geq 65(\mathrm{y})$ & 85 & 46.2 & 34 & 34.7 & 51 & 59.3 & $<0.001$ \\
\hline \multicolumn{8}{|l|}{${ }^{\mathrm{b}}$ Gender } \\
\hline Female & 67 & 36.4 & 34 & 34.7 & 33 & 38.4 & \multirow[t]{2}{*}{0.605} \\
\hline Male & 117 & 63.6 & 64 & 65.3 & 53 & 61.6 & \\
\hline${ }^{\mathrm{a}} \mathrm{BMI}\left(\mathrm{kg} / \mathrm{m}^{2}\right)$ & 30.4 & 8.0 & 29.8 & 7.2 & 31.1 & 8.8 & 0.271 \\
\hline \multicolumn{8}{|l|}{${ }^{\mathrm{b}}$ Ethnicity } \\
\hline Caucasian & 100 & 54.3 & 52 & 53.1 & 48 & 55.8 & \multirow[t]{5}{*}{0.495} \\
\hline Black & 12 & 6.5 & 5 & 5.1 & 7 & 8.1 & \\
\hline Hispanic & 48 & 26.1 & 30 & 30.6 & 18 & 20.9 & \\
\hline Asian & 18 & 9.8 & 9 & 9.2 & 9 & 10.5 & \\
\hline Other & 6 & 3.3 & 2 & 2.0 & 4 & 4.7 & \\
\hline \multicolumn{8}{|l|}{${ }^{\mathrm{b}}$ Type of residence } \\
\hline Private home & 148 & 80.4 & 82 & 83.7 & 66 & 76.7 & \multirow[t]{3}{*}{0.214} \\
\hline Nursing home & 32 & 17.4 & 13 & 13.3 & 19 & 22.1 & \\
\hline Other & 4 & 2.2 & 3 & 3.1 & 1 & 1.2 & \\
\hline \multicolumn{8}{|l|}{ bocial history } \\
\hline Smoking history & 56 & 30.4 & 24 & 24.5 & 32 & 37.2 & 0.061 \\
\hline Alcohol use & 58 & 31.5 & 34 & 34.7 & 24 & 27.9 & 0.278 \\
\hline Recreational drugs & 3 & 1.6 & 1 & 1.0 & 2 & 2.3 & 0.497 \\
\hline${ }^{\mathrm{a}}$ Hospitalization length (d) & 7 & $0-68$ & 6 & $0-68$ & 9 & $0-63$ & 0.439 \\
\hline \multicolumn{8}{|l|}{${ }^{\mathrm{b}}$ Level of care } \\
\hline Inpatient care & 79 & 42.9 & 67 & 68.4 & 12 & 14.0 & \multirow[t]{2}{*}{$<0.001$} \\
\hline Intensive care unit & 105 & 57.1 & 31 & 31.6 & 74 & 86.0 & \\
\hline \multicolumn{8}{|l|}{${ }^{\mathrm{b}}$ Comorbidities } \\
\hline Hypertension & 99 & 53.8 & 40 & 40.8 & 59 & 68.6 & $<0.001$ \\
\hline Hyperlipidemia & 71 & 38.6 & 32 & 32.7 & 39 & 45.3 & 0.078 \\
\hline Diabetes & 75 & 40.8 & 29 & 29.6 & 46 & 53.5 & 0.001 \\
\hline Coronary disease & 21 & 11.4 & 6 & 6.1 & 15 & 17.4 & 0.016 \\
\hline Heart failure & 18 & 9.8 & 6 & 6.1 & 12 & 14.0 & 0.074 \\
\hline CVA & 12 & 6.5 & 3 & 3.1 & 9 & 10.5 & 0.042 \\
\hline CKD & 19 & 10.3 & 8 & 8.2 & 11 & 12.8 & 0.303 \\
\hline Atrial fibrillation & 23 & 12.5 & 8 & 8.2 & 15 & 17.4 & 0.058 \\
\hline COPD & 15 & 8.2 & 6 & 6.1 & 9 & 10.5 & 0.283 \\
\hline Cancer & 20 & 10.9 & 6 & 6.1 & 14 & 16.3 & 0.027 \\
\hline VTE & 9 & 4.9 & 4 & 4.1 & 5 & 5.8 & 0.587 \\
\hline \multicolumn{8}{|l|}{ bVTE risk assessment models } \\
\hline Caprini high risk & 128 & 69.6 & 45 & 45.9 & 83 & 96.5 & $<0.001$ \\
\hline $\begin{array}{l}\text { IMPROVE moderate } \\
\text { VTE risk }\end{array}$ & 39 & 21.2 & 13 & 13.3 & 26 & 30.2 & $<0.001$ \\
\hline $\begin{array}{l}\text { IMPROVE high } \\
\text { VTE risk }\end{array}$ & 72 & 39.1 & 23 & 23.5 & 49 & 57.0 & $<0.001$ \\
\hline
\end{tabular}


Table 1 (Continued)

\begin{tabular}{|c|c|c|c|c|c|c|c|}
\hline \multirow[t]{2}{*}{ Variables } & \multicolumn{2}{|c|}{ Cohort $(n=184)$} & \multicolumn{2}{|c|}{ Alive $(n=98)$} & \multicolumn{2}{|c|}{ Deceased $(n=86)$} & \multirow[t]{2}{*}{${ }^{c} p$-Value } \\
\hline & $\begin{array}{l}\text { Mean/ } \\
\text { median/n }\end{array}$ & $\mathrm{SD} / \mathrm{IQR} / \%$ & $\begin{array}{l}\text { Mean/ } \\
\text { median/n }\end{array}$ & $\mathrm{SD} / \mathrm{IQR} / \%$ & $\begin{array}{l}\text { Mean/ } \\
\text { median/n }\end{array}$ & $\mathrm{SD} / \mathrm{IQR} / \%$ & \\
\hline \multicolumn{8}{|l|}{${ }^{\mathrm{b}}$ Thrombotic events } \\
\hline DVT & 5 & 2.7 & 3 & 3.1 & 2 & 2.3 & \multirow[t]{4}{*}{0.067} \\
\hline Pulmonary embolism & 9 & 4.9 & 4 & 4.1 & 5 & 5.8 & \\
\hline DIC & 12 & 6.5 & 3 & 3.1 & 9 & 10.5 & \\
\hline Stroke & 1 & 0.5 & 0 & 0 & 1 & 1.2 & \\
\hline
\end{tabular}

Abbreviations: BMI, body mass index; CKD, chronic kidney disease; COPD, chronic obstructive pulmonary disease; COVID-19, novel coronavirus disease 2019; CVA, cerebrovascular accident; DIC, disseminated intravascular coagulation; DVT, deep vein thrombosis; IMPROVE, the International Medical Prevention Registry on Venous Thromboembolism; IQR, interquartile range; SD, standard deviation; VTE, venous thromboembolism. ${ }^{a}$ Normally distributed continuous variables, presented as mean with standard deviation or median and interquartile range.

${ }^{\mathrm{b} C a t e g o r i c a l ~ v a r i a b l e s, ~ p r e s e n t e d ~ a s ~ n u m b e r ~ a n d ~ p e r c e n t a g e ~ o f ~ p a t i e n t s . ~}$

${ }^{c} p$-Value obtained with Student's t-test for continuous variables, Chi-square test or Fisher's exact test for categorical variables.

Table 2 Laboratory values during hospitalization for COVID-19 critically ill patients, NorthShore University Health System

\begin{tabular}{|c|c|c|c|c|}
\hline Variables & Cohort & Alive & Deceased & ${ }^{c} p$-Value \\
\hline${ }^{\mathrm{b}} \mathrm{Hgb}(\mathrm{gr} / \mathrm{dL})$ & 12.2 & 12.9 & 11.5 & $<0.001$ \\
\hline${ }^{\mathrm{b}}$ Neutrophil count $\left(10^{9} / \mathrm{L}\right)$ & 8.2 & 7.8 & 8.7 & 0.087 \\
\hline bymphocyte count $\left(10^{9} / \mathrm{L}\right)$ & 1.2 & 1.0 & 1.6 & 0.035 \\
\hline bPlatelet count $\left(10^{9} / \mathrm{L}\right)$ & 231.3 & 240.5 & 220.9 & 0.206 \\
\hline${ }^{\mathrm{b}}$ Fibrinogen (mg/dL) & 683.1 & 704.6 & 669.3 & 0.950 \\
\hline bPT (s) & 14.5 & 13.0 & 15.9 & $<0.001$ \\
\hline bINR & 1.4 & 1.2 & 1.6 & $<0.001$ \\
\hline${ }^{\mathrm{b}} \mathrm{D}$-dimer $(\mathrm{mg} / \mathrm{L})$ & 7.8 & 5.3 & 9.7 & $<0.001$ \\
\hline${ }^{\mathrm{b}}$ Creatinine level (mg/dL) & 1.6 & 1.0 & 2.2 & $<0.001$ \\
\hline${ }^{\mathrm{b}}$ Ferritin (ng/mL) & 2454 & 1489 & 2951 & 0.570 \\
\hline${ }^{\mathrm{b}} \mathrm{C}$-reactive protein $(\mathrm{mg} / \mathrm{dL})$ & 184.2 & 146.7 & 225.3 & $<0.001$ \\
\hline${ }^{\mathrm{b}} \mathrm{LDH}(\mathrm{U} / \mathrm{L})$ & 567.2 & 479.7 & 607.9 & 0.114 \\
\hline${ }^{\mathrm{b}} \mathrm{AST}(\mathrm{U} / \mathrm{L})$ & 186.2 & 52.2 & 283.3 & 0.002 \\
\hline${ }^{\mathrm{b}} \mathrm{ALT}(\mathrm{U} / \mathrm{L})$ & 74.4 & 47.8 & 94.5 & 0.886 \\
\hline${ }^{a}$ Albumin $(\mathrm{mg} / \mathrm{dL})$ & 2.3 & 2.7 & 2.0 & $<0.001$ \\
\hline
\end{tabular}

Abbreviations: ALT, alanine transaminase; AST, aspartate transaminase; Hgb, hemoglobin; INR, international normalized ratio; LDH, lactate dehydrogenase; PT, prothrombin time.

a Parametric continuous variable.

${ }^{\mathrm{b}}$ Nonparametric continuous variable.

' $p$-Values obtained with Student's $t$-test for parametric continuous variables; Wilcoxon's rank-sum test and Mann-Whitney U-test were used for nonparametric continuous variables.

$(\mathrm{HR}=6.50 ; 95 \% \mathrm{CI}: 2.72-15.53 ; p<0.001)$ and "High risk for VTE" by Caprini score (HR=11.507; 95\% CI: 2.697-49.104; $p=0.001$ ). Patients, classified as "Moderate risk for VTE" by IMPROVE score, did not statistically associate with thrombotic event occurrence $(\mathrm{HR}=2.82 ; 95 \% \mathrm{CI}$ : 0.62-12.75; $p=0.177$ )

The ROC curve for thrombotic event prediction with the IMPROVE score had an AUC of $0.718(p<0.001)$ with corresponding $85 \%$ sensitivity and $44 \%$ specificity for those classified as "High risk for VTE" (-Fig. 1B). The cumulative incidence of VTE for those at "Low risk for VTE" was 5.5\%, "Moderate risk for VTE" was 7.7\%, and "High risk for VTE" was 27.8\% (-Fig. 2B).
The ROC curve for thrombotic event prediction with the Caprini score had an AUC of $0.722(p<0.001)$ with corresponding $92 \%$ sensitivity and 35\% specificity for the designated dichotomized cut-off ( - Fig. 3B). When stratified by Caprini scores, the cumulative incidence of VTE increased in a linear fashion with increasing score. Patients classified at very low risk (Caprini score: 0-2) had no VTE events. Those stratified at moderate risk (Caprini score: 3-4) had a VTE incidence of $4.5 \%$, high risk (Caprini score: 5-6) had a VTE incidence of $7.4 \%$, very high risk (Caprini score 7-8) had a VTE incidence of 17.6\%, and the highest risk (Caprini score $>8$ ) had a VTE incidence of $28.0 \%$ (-Fig. 4B). 

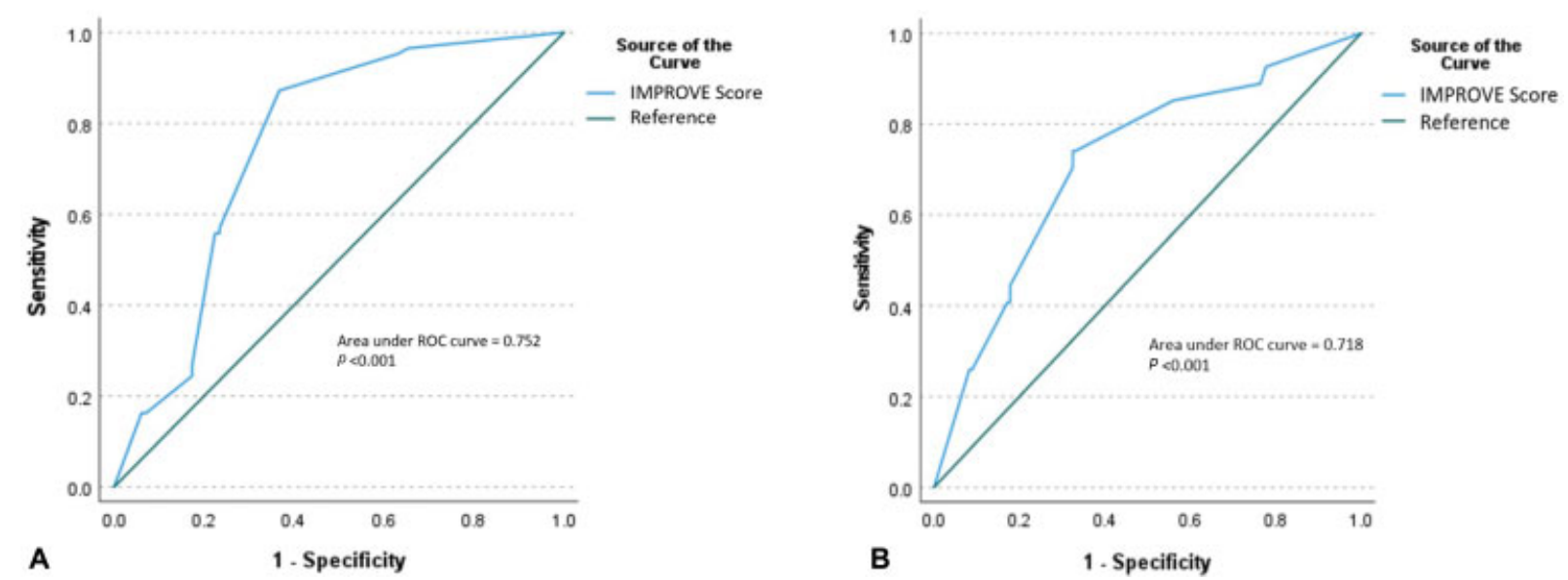

Fig. 1 (A) Receiver-operating characteristics (ROC) curve of IMPROVE score for prediction of mortality. (B) Receiver-operating characteristics curve of IMPROVE score for prediction of thrombotic events. IMPROVE, the International Medical Prevention Registry on Venous Thromboembolism.
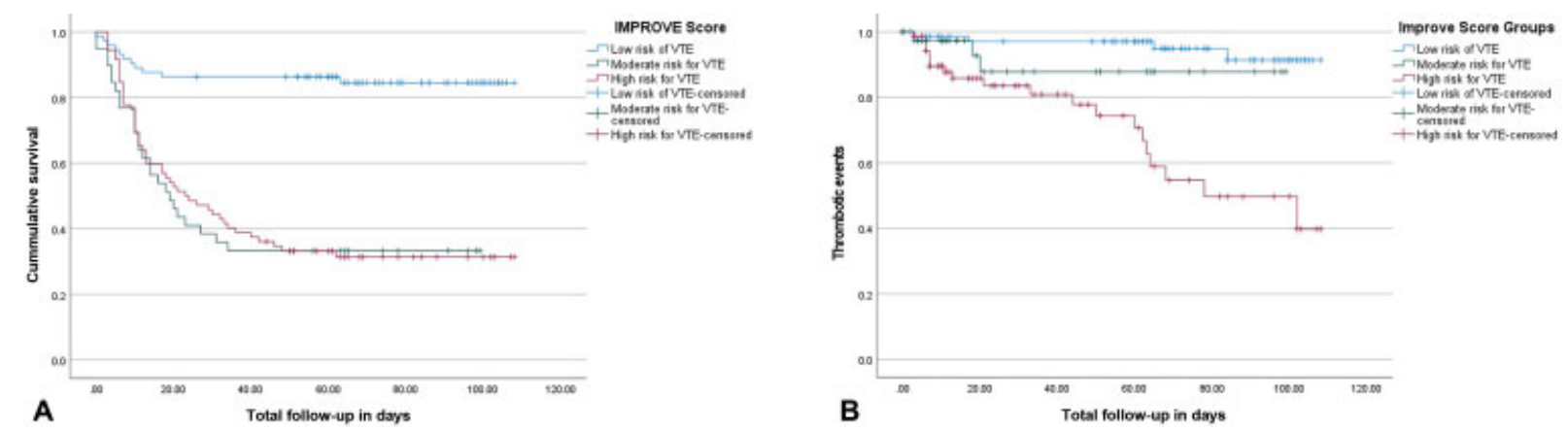

Fig. 2 (A) Kaplan-Meier curve demonstrating survival of different groups by IMPROVE score. (B) Kaplan-Meier curve demonstrating cumulative thrombotic event survival of different groups by IMPROVE score. IMPROVE, the International Medical Prevention Registry on Venous Thromboembolism; VTE, venous thromboembolism.
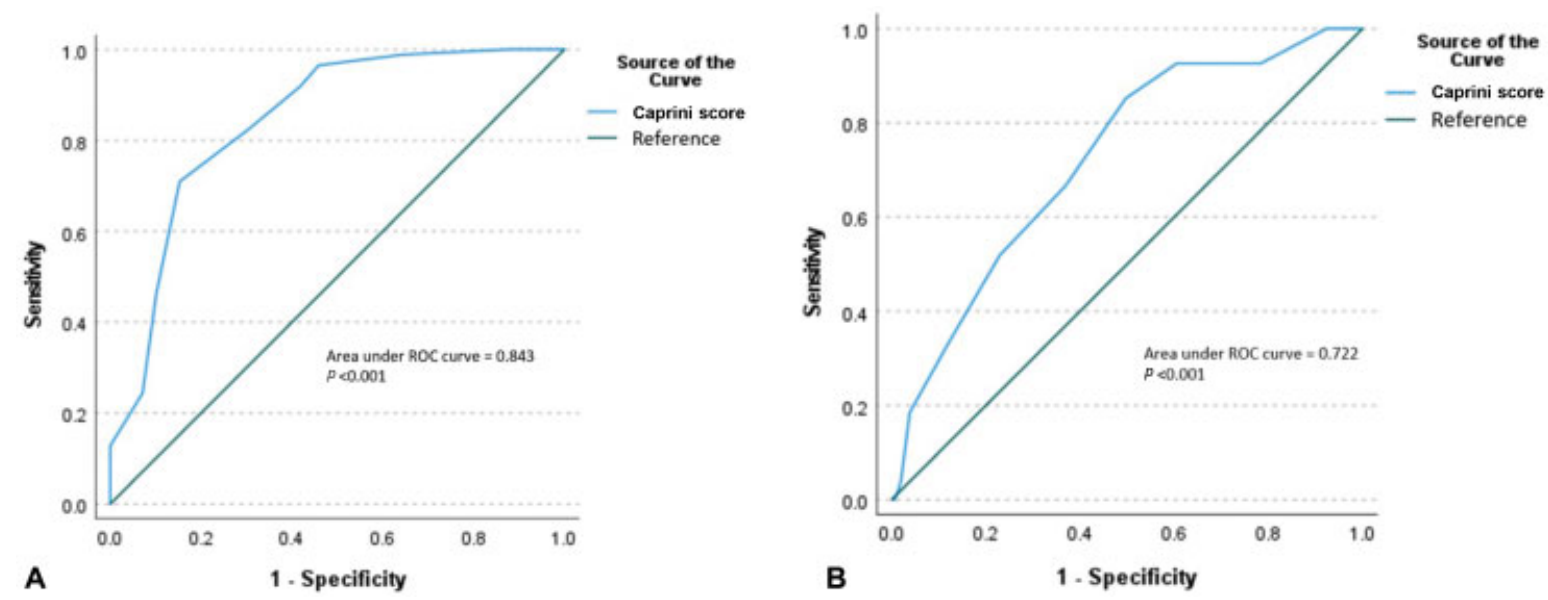

Fig. 3 (A) Receiver-operating characteristics curve (ROC) of Caprini score for prediction of mortality. (B) Receiver-operating characteristics curve of Caprini score for prediction of thrombotic events.

\section{Discussion}

In our cohort of hospitalized patients with severe COVID-19 disease, the IMPROVE and Caprini risk assessment models were both strong and independent predictors of mortality. Similarly, both scores were strong and independent predictors of thrombotic event occurrence in those classified as "High risk for VTE" by either RAM. The high mortality seen in our patients with severe COVID-19 disease is similar to prior reports. ${ }^{4}$

Severe COVID-19 is associated with dysregulation of the coagulation system, high incidence of arterial and venous thrombosis in large vessels and microvascular beds, and 

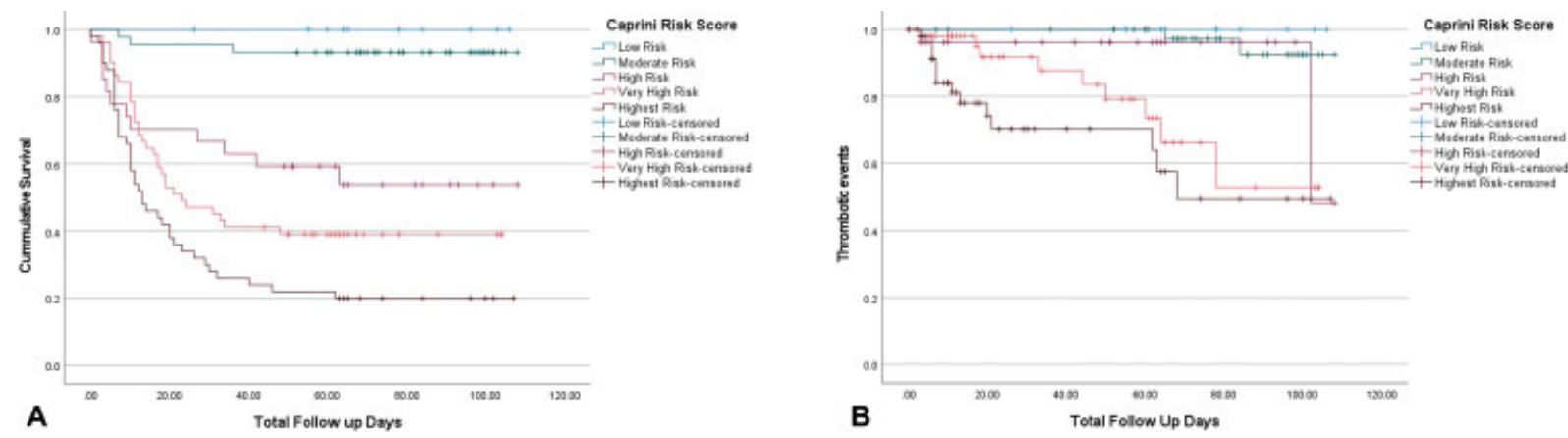

Fig. 4 (A) Kaplan-Meier curve demonstrating survival of different groups by Caprini score. (B) Kaplan-Meier curve demonstrating cumulative thrombotic event survival of different groups by Caprini score.

associated poor prognosis. ${ }^{20}$ This in part, is thought to be a consequence of the dysregulated immune response leading to endothelial dysfunction, increased vascular permeability, and intrinsic thrombophilia. ${ }^{21}$ With this in mind, we chose to evaluate the performance of two well-validated VTE scores and challenged their predictive capacity for the hard outcome of mortality in multivariate analysis. We found that patients classified as "Moderate or High risk for VTE" by the IMPROVE RAM or "High risk for VTE" by the Caprini RAM, had significantly higher mortality. Similarly, patients classified as "High risk for VTE" by the IMPROVE score and "High risk for VTE" by Caprini score had a significantly higher number of thrombotic events.

The Caprini risk score is a widely validated RAM that weights independent risk factors for the individual summing up a total score that correlates with the VTE risk. Importantly, it uses data readily available from the patient's history and physical examination without the inclusion of laboratory or imaging data. ${ }^{22}$ The IMPROVE VTE RAM is a widely accepted and validated score calibrated in acutely ill medical patients. It encompasses seven independent VTE risk factors in a hospital setting and does not require additional imaging or laboratory information to be completed. ${ }^{16}$ In the setting of a global pandemic, placing health care systems under significant constraint, the application of a clinical score that uses readily obtainable data can be a practical strategy for triaging individuals. If limited availability of medical personnel is a limiting factor, patient friendly forms are available in multiple languages and have an excellent correlation with physician-driven forms..$^{23,24}$

As the COVID-19 pandemic continues to spread around the globe, there is a better understanding of its clinical and epidemiological behavior. Although most patients will have a mild form of the disease, there is a nonnegligible $5 \%$ of patients who progress into severe illness, with reported mortality ranging from 49 to $61.5 \%{ }^{4,25}$ In our cohort of patients with severe disease, mortality was comparable $(46.7 \%)$ to the reported literature. Similarly, the comparison of comorbidities between deceased and survivors revealed significantly older age, and the presence of cardiovascular risk factors and malignancy in those who died, as previously reported. ${ }^{9,26,27}$

One of the challenges that continue to burden health care systems around the globe is the adequate allocation of resources when a large number of patients is in need in a given time.
To alleviate this burden, efficient diagnosis and resource utilization are prioritized, and reliable prognostic information as provided by RAM could be a determinant for behavioral change.

Several risk-scoring models have been developed and a few internally validated to help prioritize high-risk individuals with COVID-19. ${ }^{28-32}$ All these scores rely on clinical data, laboratory data, and imaging features to generate their prediction. It is important to note that at the time of our submission, these original documents were not peer-reviewed nor have undergone the rigors of external validation, and optimization could have occurred before their publication. Furthermore, in a systematic review of the existing prediction models for diagnosis and prognosis of COVID-19, Wynants et al and the COVIDPRECISE (Precise Risk Estimation to optimise covid-19 Care for Infected or Suspected patients in diverse settings) group group rendered the existing models at high risk of bias after independent data extraction using the Critical Appraisal and Data Extraction for Systematic Reviews of Prediction Modeling Studies (CHARMS) checklist and prediction model risk of bias assessment tool (PROBAST). ${ }^{10}$

Following hospitalization for medical illness, extended thromboprophylaxis (ET) with LMWH, or direct oral anticoagulants (DOACs) can reduce the risk of VTE at the cost of increased bleeding, with net clinical benefit seen by the DOAC rivaroxaban in key low bleed risk subgroups of medically ill patients. ${ }^{17,33-36}$ Currently, the decision to proceed with ET is largely based on individualized risk stratification for VTE and balancing the patients' bleeding risk. This practice is advised in patients with COVID-19 by the current consensus. ${ }^{37}$

\section{Strengths and Limitations}

Our analysis showed the strong predictive ability of both VTE risk assessment models for the hard outcome of mortality in this cohort of patients with severe COVID-19. It also validates the predicting capacity for thrombotic events in this population at increased risk. Because routine screening for VTE or autopsies was not performed routinely amid the pandemic, we could not verify if deaths were VTE related. Also, given the initial scarcity of personal protective equipment that was seen nationwide, is likely that the true number of thrombotic events was underestimated.

Despite the strengths of our findings, there are limitations intrinsic to the observational design of our study and the 
relatively small sample size from a single-center experience conferring a degree of ascertainment bias. Furthermore, the severity of disease from COVID-19 in our cohort introduces selection bias, thus the results may not be generalizable to all comers with COVID-19 disease.

Like many other retrospective studies, we did not perform a formal power calculation. Therefore, our study might be underpowered to draw firm conclusions about the association of the VTE risk tools, mortality, and VTE. Hence, external validation with large prospective cohorts should be pursued to confirm our findings.

Nonetheless, the implementation of either VTE RAM may be a valuable source with prognostic value for the outcome of death or thrombosis. With further validation, this strategy can assist in triaging individuals with severe COVID-19 disease and optimize resource allocation.

\section{Conclusion}

The IMPROVE and Caprini VTE risk assessment models were independent predictors of mortality and thrombotic events in our cohort of patients with severe COVID-19. With largescale validation, this prognostic information can assist triaging individuals and optimize resource allocation.

\section{Authors' Contributions}

L.H.P.R, A.T., A.C.S., J.C., and A.P. conceived of the presented idea. L.H.P.R, I.M., E.K., A.N., A.A., E.L., V.M., and K.R.B. abstracted the patient data. L.H.P.R. lead the team and performed the statistical analysis. A.T. and A.C.S. verified the analytical methods and supervised the findings. All authors discussed the results and contributed to the final manuscript.

\section{Conflict of Interest}

1. L.H.P.R. has received research support from Biotap.

2. A.C.S. is a consultant for Boehringer Ingelheim, Janssen, BMS, Portola, Bayer, the ATLAS Group. Has received research funds from Boehringer Ingelheim and Janssen.

3. J.C. is a consultant for Recovery Force. Part of the Steering Committee of Janssen R\&D. Bleeding Advisory Board for Pfizer, Advisory board of BMS and Alexion Pharmaceuticals. Has received honoraria from Sanofi and Arjo, Inc.

4. A.T. has received research support from Biotap, BMS, Doasense. Research and educational grant from Janssen R\&D. Is a consultant for Recovery Force.

\section{References}

1 Huang C, Wang Y, Li X, et al. Clinical features of patients infected with 2019 novel coronavirus in Wuhan, China. Lancet 2020;395 (10223):497-506

2 https://www.who.int/publications/m/item/weekly-epidemiological-update-15-december-2020. 2020

3 Bi Q Wu Y, Mei S, et al. Epidemiology and transmission of COVID19 in 391 cases and 1286 of their close contacts in Shenzhen, China: a retrospective cohort study. Lancet Infect Dis 2020;20 (08):911-919
$4 \mathrm{Wu} \mathrm{Z,} \mathrm{McGoogan} \mathrm{JM.} \mathrm{Characteristics} \mathrm{of} \mathrm{and} \mathrm{important} \mathrm{lessons}$ from the coronavirus disease 2019 (COVID-19) outbreak in China: summary of a report of 72314 cases from the chinese center for disease control and prevention. JAMA 2020;323(13):1239-1242

5 Rogers MA, Levine DA, Blumberg N, Flanders SA, Chopra V, Langa KM. Triggers of hospitalization for venous thromboembolism. Circulation 2012;125(17):2092-2099

6 Tang N, Li D, Wang X, Sun Z. Abnormal coagulation parameters are associated with poor prognosis in patients with novel coronavirus pneumonia. J Thromb Haemost 2020;18(04):844-847

7 Fan BE, Chong VCL, Chan SSW, et al. Hematologic parameters in patients with COVID-19 infection. Am J Hematol 2020;95(06):E131-E134

8 Zhou F, Yu T, Du R, et al. Clinical course and risk factors for mortality of adult inpatients with COVID-19 in Wuhan, China: a retrospective cohort study. Lancet 2020;395(10229):1054-1062

9 Grasselli G, Zangrillo A, Zanella A, et al. Baseline characteristics and outcomes of 1591 patients infected with SARS-CoV-2 admitted to ICUs of the Lombardy Region, Italy. JAMA 2020;323(16):1574-1581

10 Wynants L, Van Calster B, Collins GS, et al. Prediction models for diagnosis and prognosis of covid-19 infection: systematic review and critical appraisal. BMJ 2020;369:m1328

11 Wang T, Chen R, Liu C, et al. Attention should be paid to venous thromboembolism prophylaxis in the management of COVID-19. Lancet Haematol 2020;7(05):e362-e363

12 Rosenberg D, Eichorn A, Alarcon M, McCullagh L, McGinn T, Spyropoulos AC. External validation of the risk assessment model of the International Medical Prevention Registry on Venous Thromboembolism (IMPROVE) for medical patients in a tertiary health system. J Am Heart Assoc 2014;3(06):e001152

13 Caprini JA, Arcelus JI, Reyna JJ. Effective risk stratification of surgical and nonsurgical patients for venous thromboembolic disease. Semin Hematol 2001;38(02, Suppl 5):12-19

14 Cohoon KP, Mahé G, Tafur AJ, Spyropoulos AC. Emergence of institutional antithrombotic protocols for coronavirus 2019. Res Pract Thromb Haemost 2020;4(04):510-517

15 Wisniewski MF, Kieszkowski P, Zagorski BM, Trick WE, Sommers M, Weinstein RA. Development of a clinical data warehouse for hospital infection control. J Am Med Inform Assoc 2003;10(05):454-462

16 Spyropoulos AC, Anderson FA Jr, FitzGerald G, et al; IMPROVE Investigators. Predictive and associative models to identify hospitalized medical patients at risk for VTE. Chest 2011;140(03):706-714

17 Spyropoulos AC, Ageno W, Albers GW, et al; MARINER Investigators. Rivaroxaban for thromboprophylaxis after hospitalization for medical Illness. N Engl J Med 2018;379(12):1118-1127

18 Shuman AG, Hu HM, Pannucci CJ, Jackson CR, Bradford CR, Bahl V. Stratifying the risk of venous thromboembolism in otolaryngology. Otolaryngol Head Neck Surg 2012;146(05):719-724

19 Bahl V, Hu HM, Henke PK, Wakefield TW, Campbell DA Jr., Caprini JA. A validation study of a retrospective venous thromboembolism risk scoring method. Ann Surg 2010;251(02):344-350

20 Klok FA, Kruip MJHA, van der Meer NJM, et al. Incidence of thrombotic complications in critically ill ICU patients with COVID-19. Thromb Res 2020;191:145-147

21 Walborn A, Rondina M, Mosier M, Fareed J, Hoppensteadt D. Endothelial dysfunction is associated with mortality and severity of coagulopathy in patients with sepsis and disseminated intravascular coagulation. Clin Appl Thromb Hemost 2019;25:1076029619852163

22 Caprini JA. Thrombosis risk assessment as a guide to quality patient care. Dis Mon 2005;51(2,3):70-78

23 Fuentes HE, Paz LH, Al-Ogaili A, et al. Validation of a patientcompleted Caprini risk score for venous thromboembolism risk assessment. TH Open 2017;1(02):e106-e112

24 Paz Rios LH, Fuentes HE, Oramas DM, et al. Validation of a patientcompleted Caprini risk assessment tool for Spanish, Arabic, and Polish Speakers. Clin Appl Thromb Hemost 2018;24(03):502-512

$25 \mathrm{Xu}$ J, Yang X, Yang L, et al. Clinical course and predictors of 60-day mortality in 239 critically ill patients with COVID-19: a multicenter retrospective study from Wuhan, China. Crit Care 2020;24(01):394 
26 Xie J, Tong Z, Guan X, Du B, Qiu H. Clinical characteristics of patients who died of coronavirus disease 2019 in China. JAMA Netw Open 2020;3(04):e205619

27 Bhatraju PK, Ghassemieh BJ, Nichols M, et al. Covid-19 in critically ill patients in the Seattle Region - case series. N Engl J Med 2020; 382(21):2012-2022

28 Yan LZH-T, Xiao Y, et al. Prediction of criticality in patients with severe Covid-19 infection using three clinical features: a machine learning-based prognostic model with clinical data in Wuhan. medRxiv 2020. Doi: 101101/2020022720028027

29 Sarkar JCP. A machine learning model reveals older age and delayed hospitalization as predictors of mortality in patients with covid-19. medRxiv 2020. Doi: 101101/2020032520043331

30 Pourhomayoun MSM. Predicting mortality risk in patients with covid-19 using artificial intelligence to help medical decisionmaking. medRxiv 2020. Doi: 101101/2020033020047308

31 Levy TJRS, Coppa K, et al. Estimating survival of hospitalized covid-19 patients from admission information. medRxiv 2020. Doi: $101101 / 2020042220075416$

32 Singh KVT, Tang S, et al. Validating a widely implemented deterioration index model among hospitalized covid-19 patients. medRxiv 2020. Doi: 101101/2020042420079012
33 Hull RD, Schellong SM, Tapson VF, et al; EXCLAIM (Extended Prophylaxis for Venous ThromboEmbolism in Acutely Ill Medical Patients With Prolonged Immobilization) study. Extended-duration venous thromboembolism prophylaxis in acutely ill medical patients with recently reduced mobility: a randomized trial. Ann Intern Med 2010;153(01):8-18

34 Cohen AT, Harrington RA, Goldhaber SZ, et al; APEX Investigators. Extended thromboprophylaxis with betrixaban in acutely ill medical patients. N Engl J Med 2016;375(06):534-544

35 Cohen AT, Spiro TE, Büller HR, et al; MAGELLAN Investigators. Rivaroxaban for thromboprophylaxis in acutely ill medical patients. N Engl J Med 2013;368(06):513-523

36 Spyropoulos AC, Lipardi C, Xu J, et al. Improved benefit risk profile of rivaroxaban in a subpopulation of the MAGELLAN study. Clin Appl Thromb Hemost 2019;25:1076029619886022

37 Bikdeli B, Madhavan MV, Jimenez D, et al; Global COVID-19 Thrombosis Collaborative Group, Endorsed by the ISTH, NATF, ESVM, and the IUA, Supported by the ESC Working Group on Pulmonary Circulation and Right Ventricular Function. COVID-19 and thrombotic or thromboembolic disease: implications for prevention, antithrombotic therapy, and follow-up: JACC stateof-the-art review. J Am Coll Cardiol 2020;75(23):2950-2973 